hep-th/0208126

EFI-02-98

\title{
Holography and String Dynamics in Time-dependent Backgrounds
}

\author{
Akikazu Hashimoto at and Savdeep Sethi ${ }^{a, b[}$ \\ ${ }^{a}$ School of Natural Sciences \\ Institute for Advanced Study \\ Princeton, NJ 08540 \\ ${ }^{b}$ Enrico Fermi Institute \\ University of Chicago \\ Chicago, IL 60637
}

\begin{abstract}
We study the dynamics of D-branes in a smooth time-dependent background. The theory on the branes is a time-dependent non-commutative field theory. We find the metric and fluxes that determine the dual holographic closed string theory. This provides a concrete example of holography in a cosmological setting.
\end{abstract}

\footnotetext{
${ }^{1}$ e-mail: aki@ias.edu

${ }^{2}$ e-mail: sethi@theory.uchicago.edu
} 
In order to apply string theory to problems in cosmology, it is essential that we learn how to formulate string theory in backgrounds with explicit time dependence. Recently, various quotients of Minkowski space-time by groups which acts both on space and time have been studied as simple concrete realizations of string theory on time-dependent backgrounds. Generic orbifolds of space-time give rise to singularities such as closed time-like loops. These closed causal curves tend to invalidate the usual techniques of perturbative string theory because of large back-reaction. One example of a space-time orbifold which avoids these complications is the orbifold associated to the "null-brane" geometry. This quotient was first considered in [1]. String theory (and M theory) in this particular quotient background has been studied independently in [2], and also considered recently in [3, 4].

There are two particular distinguishing features of this quotient: it preserves one-half of the flat space supersymmetry, and it preserves an isometry along a light-cone direction. For these reasons, null-branes appear to provide a promising laboratory in which to explore issues of time-dependence in the simplest of settings.

For a given string theory background, it is often instructive to study the dynamics of open strings by adding D-branes. The physics of open strings can be further simplified by taking decoupling limits which freeze out massive open string modes and closed string excitations. The limiting theory for the open strings is generally non-gravitational, and can typically be formulated as a field theory. However, it is possible to show in some cases that these decoupled open strings have a dual description in terms of a theory of gravity, along the lines of the AdS/CFT correspondence.

In this paper, we will show that D-branes in the background of null-branes have an interesting decoupling limit. The decoupled theory turns out to be a novel kind of noncommutative Yang-Mills theory whose non-commutativity parameter varies as a function of the space-time coordinates. We will further show that this theory has a simple dual supergravity description. The supergravity dual is also time-dependent. As such, this noncommutative field theory can be thought of as a holographic description of a time-dependent string theory background. In this particular example, all of the time dependence of the dual supergravity background is encoded in the time dependence of the non-commutativity parameter on the field theory side. From this explicit example, we are led to the conclusion that the holographic description of more realistic cosmological space-times may very well require non-local field theory.

Let us begin by reviewing the geometry of the null-brane. This is simply an orbifold of 
3+1-dimensional Minkowski space-time

$$
d s^{2}=-2 d x^{+} d x^{-}+d x^{2}+d z^{2}
$$

by the identification

$$
\begin{aligned}
& x^{+} \sim x^{+} \\
& x \sim x+2 \pi x^{+} \\
& x^{-} \sim x^{-}+2 \pi x+\frac{1}{2}(2 \pi)^{2} x^{+} \\
& z \sim z+2 \pi R .
\end{aligned}
$$

This space can also be described in terms of the coordinates [1, 2]

$$
\hat{x}^{+}=x^{+}, \quad \hat{x}^{-}=x^{-}-\frac{z x}{R}+\frac{z^{2} x^{+}}{2 R^{2}}, \quad \hat{x}=x-\frac{z x^{+}}{R}, \quad \hat{z}=\frac{z}{R}
$$

in which the orbifold acts only on a single coordinate

$$
\hat{z} \sim \hat{z}+2 \pi
$$

The metric in these coordinates takes the form

$$
d s^{2}=-2 d \hat{x}^{+} d \hat{x}^{-}+d \hat{x}^{2}+\left(\left(\hat{x}^{+}\right)^{2}+R^{2}\right) d \hat{z}^{2}+2\left(\hat{x}^{+} d \hat{x}-\hat{x} d \hat{x}^{+}\right) d \hat{z} .
$$

Consider embedding this geometry into type IIA string theory by appending six flat additional directions. The quotient preserves the half of the supersymmetries which satisfy,

$$
\Gamma^{+} \epsilon=0 \text {. }
$$

We can now consider placing a D2-brane extended along the $x^{+}, x^{-}$, and $x$ directions, and localized in the $z$ direction. Such D2-brane preserves spinors that satisfy

$$
\epsilon^{+}=\Gamma^{0} \Gamma^{1} \Gamma^{2} \epsilon^{-}
$$

where $\epsilon^{+}$and $\epsilon^{-}$are spinors with definite chirality under $\Gamma^{0} \Gamma^{1} \cdots \Gamma^{9}$. Conditions (间) and (元) are satisfied simultaneously by 8 of the 32 supersymmetries of the type IIA theory.]

In order to extract the decoupled theory on a D2-brane, one usually scales $\alpha^{\prime}$ to zero while keeping the two-dimensional Yang-Mills coupling $g_{Y M}^{2}=g_{s} / \sqrt{\alpha^{\prime}}$ fixed.2 Here, however, there will be infinitely many copies of this D2-brane in the covering space because of the orbifolding. Therefore, we need to account for the winding modes which arise from the strings which stretch from a D2-brane to its image. One way to do this is to scale $R=\alpha^{\prime} / \widetilde{R}$

\footnotetext{
${ }^{1}$ The supersymmetry of branes in the null-brane geometry was also considered recently in 5 .

${ }^{2}$ We have ignored numerical factors of order one.
} 
keeping $\widetilde{R}$ fixed. Then, in the $\alpha^{\prime} \rightarrow 0$ limit, all but the ground state of the strings in the wound sector become infinitely massive and decouple. The surviving wound strings can then be thought of as momentum modes on a D3-brane which now wraps the dual coordinate $\widetilde{z}$ whose radius is $\widetilde{R}$.p

We have not yet stated precisely what decoupled theory actually lives on the D3-brane. In order to address this question, it is useful to define a new set of coordinates by the change of variables [6],

$$
\begin{aligned}
& x^{+}=y^{+} \\
& x=y^{+} y \\
& x^{-}=y^{-}+\frac{1}{2} y^{+} y^{2} .
\end{aligned}
$$

Unlike the transformation of (3), this change of variables is singular, but it has two merits. The quotient identification is simple,

$$
\begin{aligned}
& y^{+} \sim y^{+} \\
& y \sim y+2 \pi \\
& y^{-} \sim y^{-} \\
& z \sim z+2 \pi R
\end{aligned}
$$

and the metric is also simple:

$$
d s^{2}=-2 d y^{+} d y^{-}+\left(y^{+}\right)^{2} d y^{2}+d z^{2} .
$$

In particular, there are no off-diagonal terms involving $d z$. We observe that the D3-brane in question is equivalent to what one obtains after the following sequence of steps:

1. Start with a D3-brane wrapped on $\mathbb{R}^{1,2} \times S^{1}$ with metric

$$
d s^{2}=-2 d y^{+} d y^{-}+\left(y^{+}\right)^{2} d y^{2}+d \widetilde{z}^{2}, \quad \widetilde{z} \sim \widetilde{z}+2 \pi \widetilde{R}
$$

2. T-dualize along $\widetilde{z}$ to obtain D2-branes on $\mathbb{R}^{1,2} \times S^{1}$ localized on the $z$ circle with metric,

$$
d s^{2}=-2 d y^{+} d y^{-}+\left(y^{+}\right)^{2} d y^{2}+d z^{2}, \quad z \sim z+2 \pi R, \quad R=\frac{\alpha^{\prime}}{\widetilde{R}}
$$

3. Now "twist" the $y$ coordinate with respect to $z$ so that

$$
z \sim z+2 \pi R, \quad y \sim y+2 \pi
$$

and introduce the new coordinate:

$$
\widetilde{y}=y-\frac{z}{R}
$$

\footnotetext{
${ }^{3}$ Exchanging the M-theory circle and the T-duality circle in the type IIA description of the above construction gives rise to the S-dual theory in type IIB.
} 
4. Lastly, T-dualize back along the $z$ direction.

The sequence of steps enumerated above is a familiar one. Had the metric on $\mathbb{R}^{1,2} \times S^{1}$ been the flat metric,

$$
d s^{2}=-d t^{2}+d x^{2}+d y^{2}+d z^{2}
$$

and had we twisted the $y$ coordinate by the identification

$$
z \sim z+2 \pi R, \quad y \sim y+\frac{\Delta^{2}}{\alpha^{\prime}} R
$$

in following these steps, we would have obtained the familiar non-commutative Yang-Mills theory with []]

$$
\theta^{y z}=-\theta^{z y}=\Delta^{2}
$$

A similar procedure, where one twists the plane transverse to the brane, was used to construct non-commutative dipole theories where the dipole length is proportional to the $R$-charge 8 , 9.

In analogy, it is natural to propose that the theory on the D3-brane obtained by Tdualizing a D2-brane in the null-brane background is a non-commutative Yang-Mills theory with a $*$-product defined by

$$
f * g=f g+i \theta^{\widetilde{y} \widetilde{z}}\left(\partial_{\widetilde{y}} f \partial_{\widetilde{z}} g-\partial_{\widetilde{z}} f \partial_{\widetilde{y}} g\right)+\mathcal{O}\left(\theta^{2}\right)
$$

Since we shifted $y$ by a number of order one,

$$
1=\frac{\theta^{\tilde{y} \tilde{z}}}{\alpha^{\prime}} R=\frac{\theta^{\tilde{y} \tilde{z}}}{\widetilde{R}}
$$

So $\theta^{\tilde{y} \tilde{z}}=\widetilde{R}$.

Since the $y$ coordinates are somewhat singular compared to the $x$ coordinates, let us recast this product in terms of the original coordinates. This can be done straightforwardly. Simply map from $y$ coordinates to the $x$ coordinates using the relation (8) with $y$ replaced by $\widetilde{y}$ so that one finds

$$
\frac{\partial}{\partial \widetilde{y}}=x^{+} \frac{\partial}{\partial x}+x \frac{\partial}{\partial x^{-}}
$$

One can therefore write the $*$-product in the $x$ coordinates in the standard form

$$
f(x) * g(x)=f(x) g(x)+i \theta^{\mu \nu} \partial_{\mu} f(x) \partial_{\nu} g(x)+\mathcal{O}\left(\theta^{2}\right)
$$

\footnotetext{
${ }^{4}$ This procedure of mapping $x$ to $y$, replacing $y$ by $\widetilde{y}$, and mapping back to $x$ is what gives rise to the $\hat{x}$ coordinates used in (3).
} 
with

$$
\theta^{x z}=-\theta^{z x}=\widetilde{R} x^{+}, \quad \theta^{x^{-} z}=-\theta^{z x^{-}}=\widetilde{R} x
$$

and with all the other components of $\theta^{\mu \nu}$ vanishing. It is easy to verify that $\theta^{\mu \nu}$ obeys the identity

$$
\theta^{i l} \partial_{l} \theta^{j k}+\theta^{j l} \partial_{l} \theta^{k i}+\theta^{k l} \partial_{l} \theta^{i j}=0
$$

which ensures that the $*$-product is associative and that all of the terms higher order in $\theta$ can be generated systematically using the methods of [10, 11, 12]. What we have, therefore, is a concrete string theory realization of a non-commutative Yang-Mills theory whose noncommutativity parameter varies over space-time.

In the remainder of this note, we will provide two additional checks that this noncommutative Yang-Mills theory is the decoupled theory of open strings on the brane: (1) the analysis of open string variables in the brane probe approximation, and (2) the analysis of the dual supergravity solution in the sense of AdS/CFT correspondence.

To see what background the D3-brane sees, let us find the T-dual of the metric (12) with the twist (13) explicitly. Because of the twist, it is useful to use the coordinate $\widetilde{y}$ introduced in (14). In terms of $\widetilde{y}$ and $z$, the metric (12) takes the form

$$
d s^{2}=-2 d y^{+} d y^{-}+\frac{R^{2}\left(y^{+}\right)^{2}}{R^{2}+\left(y^{+}\right)^{2}} d \widetilde{y}^{2}+\frac{R^{2}+\left(y^{+}\right)^{2}}{R^{2}}\left(d z+\frac{R\left(y^{+}\right)^{2}}{R^{2}+\left(y^{+}\right)^{2}} d \widetilde{y}\right)^{2} .
$$

Using the formula for T-duality which can be found, for example, in [13, we find that the dual background is given by

$$
\begin{aligned}
d s^{2} & =-2 d y^{+} d y^{-}+\frac{R^{2}}{R^{2}+\left(y^{+}\right)^{2}}\left(\left(y^{+}\right)^{2} d \widetilde{y}^{2}+d \widetilde{z}^{2}\right) \\
B & =\frac{R\left(y^{+}\right)^{2}}{R^{2}+\left(y^{+}\right)^{2}} d \widetilde{y} \wedge d \widetilde{z} \\
e^{\phi} & =g_{s} \sqrt{\frac{R^{2}+\left(y^{+}\right)^{2}}{R^{2}}} .
\end{aligned}
$$

The metric, $B$-field, and coupling explicitly depend on $y^{+}$. However, these are the parameters natural for the closed strings which need not coincide with the parameters natural for the open strings, especially in the presence of a background $B$-field. The map between open and closed string parameters was derived in 114 for the case where the metric and $B$-fields do not vary. Although this is not the case here, let us simply apply the formulae of [14] by assuming that the background is sufficiently locally constant. The open string metric, $G$, and the closed string metric, $g$, are related by

$$
G^{\mu \nu}+i \frac{\theta^{\mu \nu}}{\alpha^{\prime}}=\left(g_{\mu \nu}+i B_{\mu \nu}\right)^{-1} .
$$


Substituting the values of $g_{\tilde{y} \tilde{y}}, g_{\tilde{z} \tilde{z}}$ and $B_{\tilde{y} \tilde{z}} \operatorname{read}$ off from (25), we find

$$
G^{\tilde{y} \tilde{y}}=\left(y^{+}\right)^{-2}, \quad G^{\tilde{z} \tilde{z}}=1, \quad \theta^{\tilde{y} \tilde{z}}=\widetilde{R} .
$$

This is in complete agreement with the form of the $*$-product anticipated in (18). Along similar lines, it is easy to show that the open string coupling

$$
G_{s}=e \sqrt{\frac{\operatorname{det}(g+B)}{\operatorname{det} g}}=g_{s}
$$

is non-varying, and sets the value of the Yang-Mills coupling constant:

$$
g_{Y M}^{2}=G_{s}
$$

We therefore conclude that although both the background metric and the coupling vary over space-time in the closed string variables, the NS-NS $B$-field also varies precisely in a manner that makes the open string metric and the coupling static. Therefore, we end up with a non-commutative Yang-Mills theory defined on a flat static background, with all of the space-time dependence absorbed into the variation of the non-commutativity length scale.

The non-commutative field theory described above is a decoupling limit of open strings on a D-brane. It is therefore natural to ask if a dual supergravity description can be found by taking the near horizon limit of the supergravity solution which describes this brane. Fortunately, because the null-brane is a simple orbifold of flat space, it turns out to be possible to construct the supergravity dual explicitly.

The near horizon geometry we seek can be constructed by following the sequence of steps involving a T-duality and a twist described earlier. In fact, the construction closely mimics the one used in 8, 16] to construct the supergravity dual of the dipole theory.

1. Start with the metric of the flat D3-brane but using the $y$ coordinates

$$
d s^{2}=f^{-1 / 2}\left(-2 d y^{+} d y^{-}+\left(y^{+}\right)^{2} d y^{2}+d \widetilde{z}^{2}\right)+f^{1 / 2}\left(d r^{2}+r^{2} d \Omega_{5}^{2}\right)
$$

where $f$ is the harmonic function of the D3-brane

$$
f=1+\frac{g N \alpha^{2}}{r^{4}}
$$

The $\widetilde{z}$ coordinate is compactified on a circle of radius $\widetilde{R}$. We will concentrate on the components of the metric parallel to the brane since the transverse components are unaffected in the following discussion.

\footnotetext{
${ }^{5}$ For theories with constant non-commutativity, this phenomena has been argued to be quite generic [15].
} 
2. T-dualize along $\widetilde{z}$. We now have the type IIA metric for D2-branes smeared along $z$,

$$
d s^{2}=f^{-1 / 2}\left(-2 d y^{+} d y^{-}+\left(y^{+}\right)^{2} d y^{2}\right)+f^{1 / 2} d z^{2} .
$$

3. Twist the $y$ coordinate so that the identification under the shift in $z$ becomes

$$
z \sim z+2 \pi R, \quad y \sim y+2 \pi
$$

Introduce a new coordinate,

$$
\widetilde{y}=y-\frac{z}{R}
$$

so that the identification of $\widetilde{y}$ is trivial

$$
z \sim z+2 \pi R, \quad \widetilde{y} \sim \widetilde{y}
$$

The metric in terms of these new variables then becomes

$$
d s^{2}=-2 f^{-1 / 2} d y^{+} d y^{-}+\frac{f^{1 / 2} R^{2}\left(y^{+}\right)^{2}}{f R^{2}+\left(y^{+}\right)^{2}} d \widetilde{y}^{2}+\frac{f R^{2}+\left(y^{+}\right)^{2}}{f^{1 / 2} R^{2}}\left(d z+\frac{R\left(y^{+}\right)^{2}}{f R^{2}+\left(y^{+}\right)^{2}} d \widetilde{y}\right)^{2} .
$$

4. T-dualize back along the $z$ direction. Applying formulae that can be found in [13] gives

$$
\begin{aligned}
d s^{2} & =f^{-1 / 2}\left(-2 d y^{+} d y^{-}+\frac{f R^{2}}{f R^{2}+\left(y^{+}\right)^{2}}\left(\left(y^{+}\right)^{2} d \widetilde{y}^{2}+d \widetilde{z}^{2}\right)\right) \\
B & =\frac{R\left(y^{+}\right)^{2}}{f R^{2}+\left(y^{+}\right)^{2}} d \widetilde{y} \wedge d \widetilde{z}
\end{aligned}
$$

for the metric and $B$-field. The dilaton and the background Ramond-Ramond field strengths can also be found by following this duality.

Now that we have found the supergravity solution (37) describing the D3-brane in the background (25), we can apply the standard near horizon scaling limit

$$
\alpha^{\prime} \rightarrow 0, \quad U=\frac{r}{\alpha^{\prime}}=\text { fixed, } \quad \widetilde{R}=\frac{\alpha^{\prime}}{R}=\text { fixed }
$$

to derive the supergravity dual of the decoupled theory on the brane. The metric in string frame is found to be

$$
d s^{2}=\alpha^{\prime}\left(\frac{U^{2}}{\sqrt{\lambda}}\left(-2 d y^{+} d y^{-}+\frac{\left(y^{+}\right)^{2} d \widetilde{y}^{2}+d \widetilde{z}^{2}}{1+\frac{\widetilde{R}^{2}\left(y^{+}\right)^{2} U^{4}}{\lambda}}\right)+\frac{\sqrt{\lambda}}{U^{2}}\left(d U^{2}+U^{2} d \Omega_{5}^{2}\right)\right),
$$

where $\lambda=g_{Y M}^{2} N$ is the 't Hooft coupling. 
Note that the form of the supergravity solution very closely resembles the supergravity dual of non-commutative Yang-Mills theory described in [17, 18]

$$
d s^{2}=\alpha^{\prime}\left(\frac{U^{2}}{\sqrt{\lambda}}\left(-d t^{2}+d x^{2}+\frac{d z^{2}+d y^{2}}{1+\frac{\Delta^{4} U^{4}}{\lambda}}\right)+\frac{\sqrt{\lambda}}{U^{2}}\left(d U^{2}+U^{2} d \Omega_{5}^{2}\right)\right)
$$

where $\Delta^{2}=\theta^{y z}$ is the non-commutativity parameter. By comparing the form of (40) and (41), we can identify the scale of non-commutativity in the $(\widetilde{y}, \widetilde{z})$-plane to be $\widetilde{R}$, which is consistent with the form of the $*$-product in (18).

The main claim of this paper is the duality between a non-commutative Yang-Mills theory with non-commutativity parameter (22) and string theory on the background (40). The background (40) is the T-dual of the null-brane geometry with large gravitational backreaction due to the presence of the D3-branes. This background inherits the explicit time-dependence of the null-brane geometry. The non-commutative field theory can therefore be interpreted as a holographic description of a time-dependent string background. It is interesting that although various fields, including the metric, dilaton, and the $B$-field, vary over space-time in the bulk description, the only space-time varying parameter on the field theory side appears to be the non-commutativity parameter.

The decoupled field theory is an interesting theory in its own right. To our knowledge, this is the first concrete string theory realization of a non-commutative field theory with non-constant non-commutativity parameter. Since the non-commutativity parameter $\theta^{\mu \nu}$ does not have an $x^{+}$component, the action will not contain higher $x^{+}$derivative terms, so it would be natural to quantize the theory treating $x^{+}$as time. It would, nonetheless, be non-trivial to quantize this theory as the action depends explicitly on $x^{+}$through the non-commutativity parameter. It would be interesting to explore the standard issues of noncommutative field theory for this kind of model, including thermodynamics, UV/IR mixing, soliton dynamics, the gauge invariant observables, and S-duality to name just a few.

The main appeal of the duality presented in this paper is that it establishes concretely the possibility of defining a cosmological background using holography. It is very interesting to speculate on the possibility of finding a holographic dual to more realistic cosmologies along similar lines. The example considered in this paper clearly demonstrates that the holographic theory, should one exist, need not be a local quantum field theory. 


\section{Acknowledgements}

The work of A. H. is supported in part by DOE Grant No. DE-FG02-90ER40542, and the Marvin L. Goldberger fellowship. The work of S. S. is supported in part by NSF CAREER Grant No. PHY-0094328, and by the Alfred P. Sloan Foundation. We would also like to thank the Aspen Center for Physics where this work was completed.

\section{References}

[1] J. Figueroa-O'Farrill and J. Simon, "Generalized supersymmetric fluxbranes," JHEP 12 (2001) 011, hep-th/0110170.

[2] D. Robbins and S. Sethi, "Strings and matrices in the null-brane space-time," to appear.

[3] H. Liu, G. Moore, and N. Seiberg, "Strings in time-dependent orbifolds," hep-th/0206182.

[4] M. Fabinger and J. McGreevy, "On smooth time-dependent orbifolds and null singularities," hep-th/0206196.

[5] J. Figueroa-O'Farrill and J. Simon, "Supersymmetric Kaluza-Klein reductions of M2 and M5 branes," hep-th/0208107.

[6] H. Liu, G. Moore, and N. Seiberg, "Strings in a time-dependent orbifold," JHEP 06 (2002) 045, hep-th/0204168.

[7] M. R. Douglas and C. M. Hull, "D-branes and the noncommutative torus," JHEP 02 (1998) 008, hep-th/9711165.

[8] A. Bergman, K. Dasgupta, O. J. Ganor, J. L. Karczmarek, and G. Rajesh, "Nonlocal field theories and their gravity duals," Phys. Rev. D65 (2002) 066005, hep-th/0103090.

[9] K. Dasgupta and M. M. Sheikh-Jabbari, "Noncommutative dipole field theories," JHEP 02 (2002) 002, hep-th/0112064.

[10] M. Kontsevich, "Deformation quantization of Poisson manifolds, I," q-alg/9709040. 
[11] A. S. Cattaneo and G. Felder, "A path integral approach to the Kontsevich quantization formula," Commun. Math. Phys. 212 (2000) 591-611, math.qa/9902090.

[12] L. Cornalba and R. Schiappa, "Nonassociative star product deformations for D-brane worldvolumes in curved backgrounds," Commun. Math. Phys. 225 (2002) 33-66, hep-th/0101219.

[13] A. Giveon, M. Porrati, and E. Rabinovici, "Target space duality in string theory," Phys. Rept. 244 (1994) 77-202, hep-th/9401139.

[14] N. Seiberg and E. Witten, "String theory and noncommutative geometry," JHEP 09 (1999) 032, hep-th/9908142.

[15] D. S. Berman et al., "Holographic noncommutativity," JHEP 05 (2001) 002, hep-th/0011282.

[16] M. Alishahiha and H. Yavartanoo, "Supergravity description of the large N noncommutative dipole field theories," JHEP 04 (2002) 031, hep-th/0202131.

[17] A. Hashimoto and N. Itzhaki, "Non-commutative Yang-Mills and the AdS/CFT correspondence," Phys. Lett. B465 (1999) 142-147, hep-th/9907166.

[18] J. M. Maldacena and J. G. Russo, "Large N limit of non-commutative gauge theories," JHEP 09 (1999) 025, hep-th/9908134. 